\title{
Electromagnetic Design of Dual Resonant Structures for Improved Sensitivity of Terahertz Label Free Bio-Sensing
}

\author{
Mihai D. Rotaru and Jan K. Sykulski, Fellow IEEE \\ School of Electronics and Computer Science, University of Southampton \\ Highfiled, Southampton, Hants, SO17 1BJ, UK \\ mr@ecs.soton.ac.uk
}

\begin{abstract}
A design is proposed exploiting full wave numerical simulation of a dual resonant structure with an aim to sense small amounts of chemical and biochemical materials. The structure is energized with free space radiation in the terahertz regime. Thanks to its asymmetric geometry two close resonances are excited. The interference between these two resonances produces a sharp change in the frequency response of the system. By selectively loading the structure with only small amounts of probe material, a relatively large shift in the frequency response may be achieved. The concept is demonstrated through simulation, while optimization of the structure and the analyte loading is attempted.
\end{abstract}

\section{INTRODUCTION}

Recent technological advances, coupled with the ability to fabricate features on substrates with nanoscale precision, have fuelled the market of biochemical sensors, with Genetic diagnostics kits - such as DNA biosensors, gene chips and lab-on-chip analyzers - developed recently. Most techniques rely on detecting the hybridization of an unknown target to a known single stranded probe DNA molecule. Contemporary hybridization detection uses fluorescent labelling, shown to be efficient, but not without drawbacks [1]. Terahertz probing has the potential for label-free detection of the DNA binding state, as several resonances in the $\mathrm{THz}$ range associated with interbackbone excitation of DNA molecules have been predicted [2], and some already explored [1,3].

Conventional terahertz spectroscopy cannot be used, as insufficient analyte is available. High sensitivity sensors employ resonant structures with frequency response shifted by dielectric loading. Solutions based on microstrip resonators, evanescent field resonances and gratings have been proposed, but they lead to complications. To reduce the system complexity and lower the cost it may be useful to separate the reader from the sensor chip, as the chip can then be considered as an expendable part. In this paper we propose a simple but efficient resonator structure that could be arranged in an array of such resonators and integrated into a chip that could be directly accessed from free space through terahertz radiation.

\section{ASYMMETRIC RESONATOR}

The resonator studied has a simple $\mathrm{X}$ shape (Fig. 1). A full wave electromagnetic simulation of the structure has been conducted when illuminated with a plane electromagnetic wave in the terahertz range. For a symmetric case, with all four arms equal, a single resonant frequency is observed at around $0.7-1.2 \mathrm{THz}$, with the resonator behaving like a moderate $\mathrm{Q}$ factor bandstop filter for waves polarized along its shorter dimension ( $y$-axis in this case). Unfortunately, the edges of this bandstop filter's response are not sufficiently sharp to achieve high sensitivity when used as a sensing element. However, when asymmetry is introduced (by reducing the length of the top two arms) a dual resonance is found, due to different parts becoming resonant at slightly different frequencies. This dual resonance has a very sharp local maximum and a zero nearby (Fig. 1) and this feature could be used for sensing purposes.

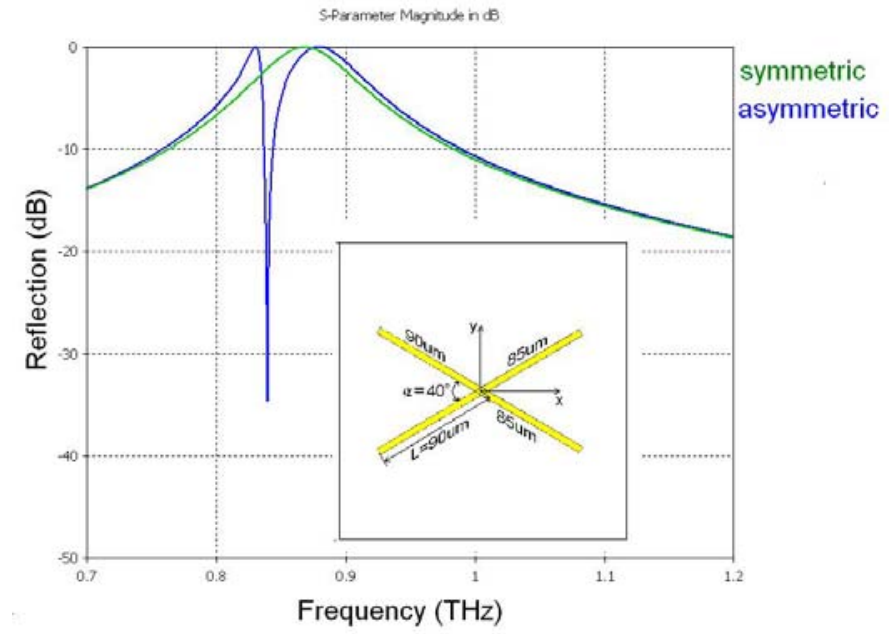

Fig. 1. Simulated variation of the reflection coefficient of the $\mathrm{X}$ resonator for an infinite array configuration: symmetric and asymmetric cases.

In our design the resonator has a thickness of $400 \mu \mathrm{m}$ and width of $5 \mu \mathrm{m}$, with the cell size $220 \mu \mathrm{m} \times 220 \mu \mathrm{m}$. Numerical simulations have been performed using CST Microwave at a unit cell level. Periodic boundary conditions in $x$ and $y$ have been applied implying the array to be infinitely large. Floquet ports with two modes are assumed for the excitation on the top and bottom surfaces of the 3D cell. A plane wave propagating normal to the $x y$ plane is modelled with mode 1 polarized in $y$ and mode 2 polarized in $x$, respectively. The model is solved using the frequency domain solver on a tetrahedral mesh. The mesh is adapted first at $1.2 \mathrm{THz}$, the highest frequency of interest, and then a number of adaptively chosen samples over the $0.7-1.2 \mathrm{THz}$ interval are solved using the same mesh. The scattering parameters $(\mathrm{S})$ are obtained from the field solution.

\section{REFERENCES}

[1] C. Debus and P. H. Bolivar, "Frequency selective surface for high sensitivity terahertz sensing," Appl. Phys. Lett. 91, 2007. 
$>11(\mathrm{f}))<$

[2] L. L. Van Zandt and V. K. Saxena, "Millimeter-microwave spectrum of DNA: Six prediction for spectroscopy," Phys. Rev. A, T, Vol. 39, Issue 5, pp. 2672-2674, March 1989.

[3] M. Nagel, M. Forst and H. Kurtz, "THz biosensing devices: fundamentals and technology," J. Phys:.Condens. Matter, Vol. 18, pp. S601-S617, 2006. 